\title{
Etude diagnostique des technologies de transformation de la pomme de cajou en jus au Bénin
}

\author{
Emmanuelle S.C.A. DEDEHOU ${ }^{1,2}$, Joseph DOSSOU ${ }^{1}$ et Mohamed M. SOUMANOU ${ }^{2 *}$ \\ ${ }^{1}$ Laboratoire de Physico-Chimie des Aliments, Département de Nutrition et Sciences Alimentaires, \\ Faculté des Sciences Agronomiques, BP 526, Cotonou, Bénin. \\ ${ }^{2}$ Unité de Recherche en Génie Enzymatique et Alimentaire, Laboratoire d'Etude et de \\ Recherche en Chimie Appliquée, Département de Génie de Technologie Alimentaire, \\ Ecole Polytechnique d'Abomey-Calavi, 01 BP 2009, Cotonou, Bénin. \\ *Auteur correspondant ; E-mail : msoumanoufr@yahoo.fr
}

\section{RESUME}

La présente étude est un diagnostic des technologies de transformation de la pomme de cajou en jus au Bénin. Suite à une pré-enquête ayant permis de connaître les zones de concentration de unités de transformation des pommes, une enquête technologique approfondie a été conduite dans un échantillon de trois unités afin de décrire les opérations unitaires de transformation et de procéder à l'analyse des caractéristiques physico-chimiques, microbiologiques et sensorielles des boissons produites. Trois types de jus de pomme de cajou ont été identifiés: le cajuina clarifié à l'amidon de manioc (J1), le cajuina clarifié au gruau de riz (J2) et le jus naturel de pomme de cajou clarifié au gruau de riz (J3). Ces trois jus se caractérisent par une bonne qualité physico-chimique, avec une acidité titrable moyenne de $0,31 \%$, un $\mathrm{pH}$ de 3,9 à 4 et des teneurs en tanin variant de 4,9 à 14,8 mg Eq de catéchine/100 ml. La teneur en vitamine $\mathrm{C}$ des jus varie de 147,0 à 185,8 mg/100 ml. Les jus ont des teneurs élevées en potassium (134,26 à 145,03 mg/100 g) contre de faibles teneurs en calcium $(2,01$ à $2,09 \mathrm{mg} / 100 \mathrm{~g})$. En dehors du $\mathrm{J} 3$ où il a été révélé quelques colonies au niveau de la flore aérobie mésophile totale, on note une absence de levures et de moisissures dans tous les jus. Au plan sensoriel, le cajuina clarifié à l'amidon de manioc (J1) et le cajuina clarifié au gruau de riz (J2) sont globalement mieux appréciés que le jus naturel de pomme de cajou clarifié au gruau de riz (J3).

(C) 2015 International Formulae Group. All rights reserved.

Mots clés: Diagnostic, jus, pomme de cajou, astringence, agents clarifiants, qualité.

\section{INTRODUCTION}

L'anacardier (Anacardium occidentale L.) est un arbre originaire du Mexique, du Pérou, du Brésil et également des Indes occidentales. Il a été naturalisé dans plusieurs pays tropicaux d'Asie et d'Afrique tels que le Vietnam, l'Inde, le Nigéria, la Tanzanie, la Côte d'Ivoire, le Mozambique et le Bénin (Michodjehoun-Mestres et al., 2009). La noix (vrai fruit) est le produit le plus important de l'anacardier. De nos jours, l'anacardier est une culture de rente en plein essor et représente pour l'Afrique une grande opportunité d'exportation de ses noix. La production mondiale a presque doublé en moins d'une décennie, passant de 2.361.384 tonnes en 2002 à 4.152 .315 tonnes en 2012 (FAO, 2014), en particulier grâce au regain d'intérêt 
de certains pays africains pour cette culture. Avec une production nationale de $170.000 \mathrm{t}$ (FAO, 2014), le Bénin est classé cinquième parmi les principaux exportateurs mondiaux de noix brute en 2012, après le Viet-Nam $(1.190 .900$ t), le Nigéria $(836.500$ t), l'Inde $(680.000 \mathrm{t})$ et la Côte d'Ivoire (450.000 t).

Certes, l'anacardier est produit de nos jours essentiellement pour sa noix, mais cet arbre fournit également la pomme (pseudo fruit) à laquelle la noix est attachée. La pomme de cajou, juteuse, a une saveur agréable et elle est généralement mise sur le marché sous forme de pulpe surgelée, de jus et de nectar (Queiroz et al., 2011). Le Brésil et l'Inde sont les deux principaux pays qui ont développé d'innombrables produits issus de la pomme de cajou et fortement ancrés dans les habitudes alimentaires de leurs populations, tandis qu'en Afrique, en dépit de la hausse exceptionnelle de la production citée cidessus, la perception très répandue selon laquelle la consommation de la pomme de cajou associée au lait occasionnerait une intoxication alimentaire mortelle (Lacroix, 2003), freine véritablement la valorisation de la pomme de cajou. Cependant, des avancées notoires dans le développement de nouveaux produits à base de cette pomme sont réalisées dans certains pays producteurs africains. L'ONG américaine International Relief Service (IRD)" a recensé et documenté dans le bassin du fleuve Gambie, en Casamance et en Guinée Bissau, près d'une cinquantaine de recettes culinaires à base de pomme de cajou (IRD, 2012). Au Bénin, divers procédés de valorisation de la pomme de cajou en jus, alcool, vin, confiture et autres produits sont aussi identifiés, dont les plus améliorés ont été élaborés et diffusés par Dossou et al. (2008). Ces auteurs ont développé plusieurs procédés de transformation de la pomme de cajou en jus, et qui sont vulgarisés de nos jours aussi bien au Bénin que dans la sous-région ouestafricaine. Certes, la transformation de la pomme de cajou en ces divers sous-produits au Bénin est une opportunité de diversification des sources de revenus des producteurs, contribuant ainsi à l'amélioration de leurs conditions de vie. Cependant, s'ils sont mal appliqués par ces producteurs surtout en milieu rural, ces procédés de transformation de la pomme de cajou pourraient affecter les caractéristiques nutritionnelles, microbiologiques et sensorielles initiales de la pomme. Or, à ce jour, la recherche scientifique ne dispose pas de connaissances élaborées, ni sur les conditions de mise en œuvre de ces procédés dans les unités de production créées, ni sur la qualité des produits obtenus. En effet, les traitements thermiques engendrent une modification de la qualité sensorielle des jus de fruit, attribuée principalement à la perte par évaporation ou par dégradation des composés volatiles responsables de l'arôme et de la saveur typique du fruit frais et/ou à la formation des composés volatiles additionnels qui ont un impact négatif sur l'arôme et la saveur du jus (Reineccius et al., 2006). On note des modifications de la composition chimique initiale des jus de pomme de cajou et des changements de couleurs, dus à la dégradation des vitamines, des caroténoïdes, des sucres, suite au traitement thermique (Damasceno et al., 2008 ; Zekpa et al., 2009 ; Sampaio et al., 2011). Par ailleurs, plusieurs auteurs affirment que la clarification pour l'élimination des tanins, décrite aussi dans les procédés de Dossou et al. (2008), constitue une étape importante dans la production du jus de pomme de cajou, qui induit également des modifications dans la composition physicochimique initiale de la pomme cajou (Campos et al., 2002 ; Couri et al., 2003 ; Jayalekshmy et John, 2004; de Abreu et al., 2005 ; Cianci et al., 2005 ; Castro et al., 2007 ; Talasila et al., 2011).

$\mathrm{Au}$ Bénin, les contraintes liées à l'utilisation de ces procédés n'étant pas connues, il est difficile de proposer des améliorations. C'est pourquoi la présente 
étude a été envisagée et porte spécifiquement sur un diagnostic des technologies de transformation de la pomme de cajou en jus au Bénin, afin d'identifier et de caractériser les opérations unitaires de ces technologies, d'en décrire les contraintes et d'envisager dans une phase ultérieure les innovations à développer en vue d'une amélioration de la production du jus de pomme de cajou.

Les différents procédés identifiés au cours de la présente étude ont été caractérisés du point de vue des opérations unitaires ainsi que des équipements utilisés et leurs impacts sur la qualité des jus obtenus ont été décrits.

\section{MATERIEL ET METHODES}

La démarche méthodologique adoptée dans la présente étude comporte trois étapes : la première étape est une recherche documentaire suivie d'une pré enquête auprès d'animateurs d'ONGs et d'organisations professionnelles agricoles des zones de production de l'anacarde, des responsables des projets/programmes intervenant dans ces zones et de certaines personnes ressources intervenant ou étant précédemment intervenues dans la filière anacarde.

Cette pré enquête a eu pour objectif d'identifier les unités de transformation de la pomme de cajou ainsi que les divers sousproduits de la pomme obtenus dans ces unités. A l'issu de cette première étape, un échantillon de trois unités de production de jus de pomme de cajou a été retenu pour une enquête technologique qui constitue la deuxième étape de cette étude. Ces unités ont été choisies du fait qu'ayant été créées depuis au moins deux ans, elles disposent d'une expérience prouvée de production et de données fiables et utiles pour la présente étude.

La troisième étape, a consisté en une caractérisation physico-chimique, microbiologique et sensorielle au laboratoire des échantillons des jus prélevés au cours de la phase d'enquête technologique.

\section{Cadre de l'étude}

L'étude a été réalisée au Centre du Bénin, dans les départements des Collines et du Borgou, reconnus comme zone très favorable à la production d'anacarde au Bénin et qui abritent aussi les plus importantes activités de transformation des noix et des pommes de cajou. Sur les dix unités de transformation de la pomme de cajou identifiées dans cette zone de l'étude, trois unités fonctionnelles de production artisanale de jus de pomme cajou ont servi de cadre pour la réalisation de l'enquête technologique. Il s'agit de l'unité Jaram de Boucousséra située dans la commune de Tchaourou entre $2^{\circ} 55^{\prime} 80^{\prime \prime}$ longitude Est et $9^{\circ} 12^{\prime} 25^{\prime \prime}$ latitude Nord (Département du Borgou), de l'unité Irédé de Savè située entre $2^{\circ} 49^{\prime} 43^{\prime \prime}$ longitude Est et $8^{\circ} 03^{\prime} 63^{\prime \prime}$ latitude Nord (Département des Collines) et de l'unité de transformation Oloré de Akpassi située dans la commune de Bantè entre $1^{\circ} 89^{\prime} 59^{\prime \prime}$ longitude Est et $9^{\circ} 12^{\prime} 25^{\prime \prime}$ latitude Nord (Département des Collines). Ces localités sont indiquées sur la carte de la Figure 1.

\section{Enquête}

L'enquête a eu pour cible les productrices de jus de pomme de cajou des différentes unités retenues. Les données ont été collectées à l'aide d'un guide d'entretien sur les aspects socioculturels de la production, les matières premières utilisées et les facteurs déterminants leur choix, la nature et la succession des opérations unitaires, les caractéristiques du matériel et les paramètres opératoires (températures, temps, masses et volumes des matières premières et des produits). L'enquête a permis d'élaborer le diagramme technologique de production du jus de pomme de cajou dans la zone de l'étude et de prélever des échantillons de jus pour les analyses de laboratoire. Pour le bilan de matière, les données de quatre essais de production relevées dans ces unités ont été considérées. 


\section{Analyses physico-chimiques}

En vue d'apprécier la qualité physicochimique et nutritionnelle des jus prélevés dans les unités enquêtées, les paramètres ciaprès ont été analysés : le $\mathrm{pH}$ a été déterminé au moyen d'un pH-mètre (JENWAY 3505 pH Meter) préalablement calibré avec des solutions tampon de $\mathrm{pH}$ 4,0 et 7,0; l'acidité titrable, exprimée en pourcentage d'acide citrique, a été déterminé par titration avec du $\mathrm{NaOH} \quad(0,1 \mathrm{~N})$ en présence de phénolphtaléine; l'Extrait sec refractométrique $\left({ }^{\circ}\right.$ Brix) a été déterminé à 20 ${ }^{\circ} \mathrm{C}$ au moyen d'un réfractomètre portatif (Refractometer FG-113 Brix/ATC 0-32\%); la clarté, la couleur, l'indice de brunissement ont été déterminés au spectrophotomètre UV (JENWAY 6715 UV/Vis Spectrophotometer) aux longueurs d'ondes respectives de $660 \mathrm{~nm}$, $440 \mathrm{~nm}, 420 \mathrm{~nm}$, avec l'utilisation de l'eau distillée comme contrôle. La teneur en tanins a été déterminée par la méthode de vanilline et exprimée en équivalent de catéchine selon Broadhurst et Jones (1978). La vitamine C a été déterminée par titration à l'iodine (AOAC, 2000) et les minéraux ont été déterminés par spectrophotométrie d'absorption atomique.

\section{Analyses microbiologiques}

La qualité microbiologique des boissons a été appréciée à travers la détermination de la flore aérobie mésophile et des levures et moisissures. Pour cela, $1 \mathrm{ml}$ de l'échantillon à analyser a été prélevé de façon aseptique auquel ont été ajoutés $9 \mathrm{ml}$ d'eau peptonée salée stérile $(5 \mathrm{~g}$ peptone, $8,5 \mathrm{~g}$ $\mathrm{NaCl}, \quad \mathrm{pH}=7,2 \pm 0,2)$; ce mélange, homogénéisé au vortex pendant 2 minutes, a servi de solution mère; des dilutions décimales successives ont été ensuite réalisées et utilisées pour l'ensemencement des boîtes de Pétri.

La flore aérobie mésophile a été déterminée sur milieu Plate Count Agar (PCA, oxoid, CM 325, Hampshire, England) ; $1 \mathrm{ml}$ de la suspension mère et de ses dilutions a été placé dans des boîtes de Pétri stériles. A ceci ont été ajoutés 10 à $15 \mathrm{ml}$ du milieu Plate Count Agar; l'ensemble a été parfaitement homogénéisé. Après solidification complète, les boîtes de Pétri ont été retournées et incubées à $30{ }^{\circ} \mathrm{C}$ pendant 72 heures. Le dénombrement des germes en Unité Formant Colonie (UFC) / ml d'échantillon des boissons analysées a été fait selon la méthode spécifiée par la norme ISO 4833: 2003.

Les levures et les moisissures ont été dénombrées suite à une incubation de $1 \mathrm{ml}$ de chaque dilution sur du Malt Extract Agar (MEA, oxoid, CM 325, Hampshire, England) à $25^{\circ} \mathrm{C}$ pendant 72 heures. Le dénombrement des germes en Unité Formant Colonie (UFC) / $\mathrm{ml}$ d'échantillon des boissons analysées a été fait selon la méthode spécifiée par la norme ISO 21527-1: 2008.

\section{Analyse sensorielle}

L'analyse sensorielle des boissons a été faite à travers un test hédonique, réalisé sur un panel de 100 dégustateurs naïs; les critères d'appréciation étant: la clarté, la couleur, le goût et l'odeur sur une échelle de 9 points allant de «extrêmement agréable (9)» à «extrêmement désagréable (1)» selon Meilgaard et al. (1987) et Sidel et al. (1993).

\section{Analyses statistiques}

Le logiciel SAS v 9.2 a servi à l'analyse statistique des données. L'analyse de la variance (ANOVA) a été faite et les différences entre les données ont été déterminées par le test de Student-NewmanKeuls au seuil de $5 \%$. 


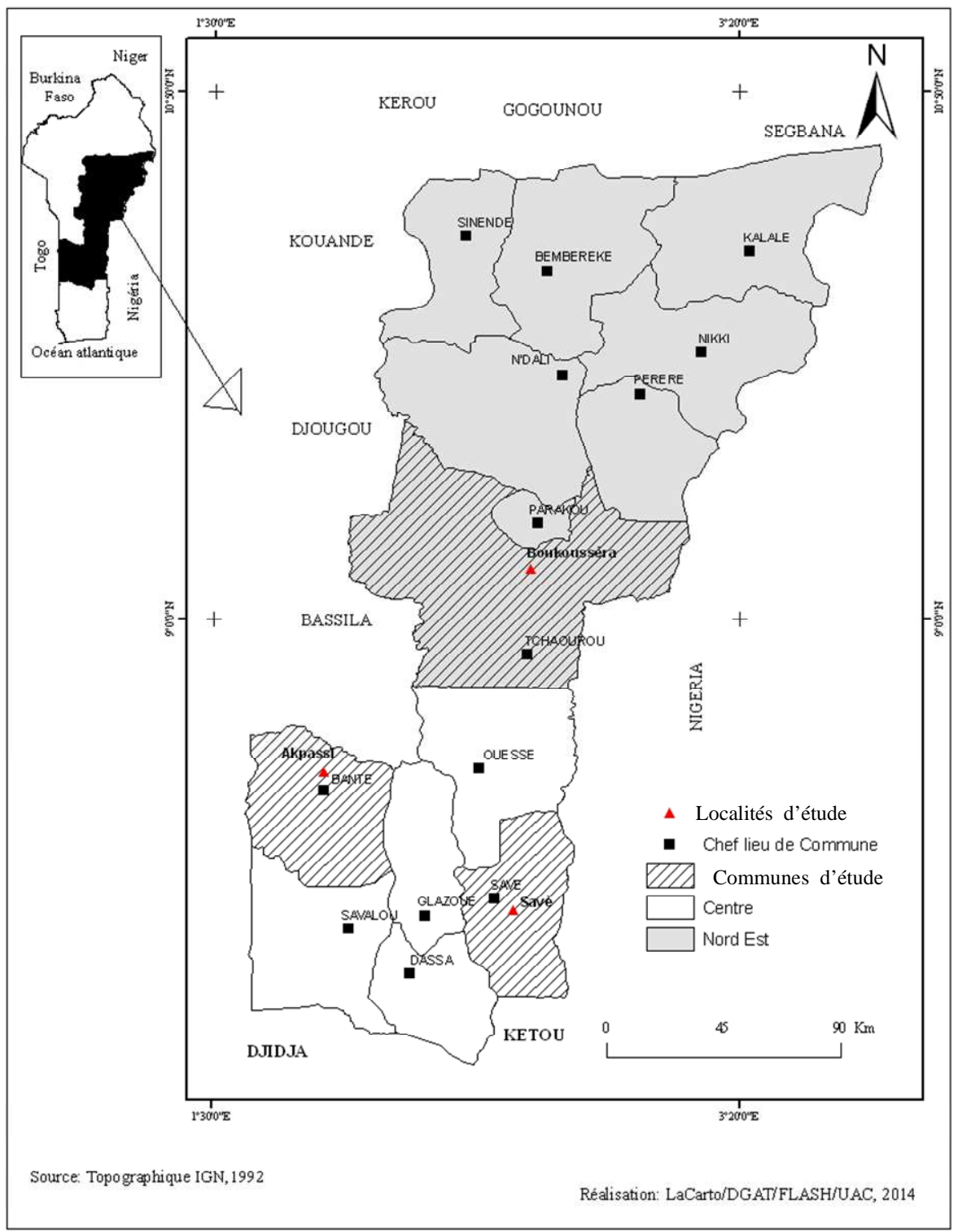

Figure 1 : Zones d'enquête de l'étude diagnostique.

\section{RESULTATS}

Caractéristiques socioculturelles de la transformation de la pomme de cajou au Bénin

Au Bénin, la pomme de cajou est initialement considérée comme un sousproduit de l'industrie de la noix de cajou. Elle est généralement abandonnée au pourrissement dans les plantations d'anacarde. En raison de son astringence, elle est très peu consommée directement comme fruit frais par les populations. A cela s'ajoute la rumeur persistante selon laquelle la consommation de la pomme fraîche serait incompatible avec certains produits comme le lait, le sucre et l'alcool à base de vin de palme, connu sous le nom de sodabi au Bénin. La pomme de cajou étant un produit hautement périssable et saisonnier, ne pouvant pas être transportée sur de longues distances, des pratiques de valorisation en alcool alimentaire et sous forme de brochettes de pommes de cajou 
séchées ont été développées par les producteurs déjà au début des années 60, lorsque l'état béninois avait envisagé la vulgarisation de l'anacardier comme culture de rente. Les autres sous-produits de la pomme de cajou recensés dans les unités enquêtées sont des jus (jus frais et cajuina). Les procédés d'obtention de ces produits sont les résultats des travaux de recherches scientifiques réalisés au Bénin.

Le jus frais ou jus naturel est l'extrait pur et brut de la pomme de cajou clarifié, mis en bouteille et stabilisé par pasteurisation. Lorsque ce jus est bien pasteurisé, à un barème modéré de $80{ }^{\circ} \mathrm{C}$ pendant 15 à $20 \mathrm{mn}$, il a un goût sucré-agréable et conserve l'arôme original de pomme de cajou. Le cajuina, par contre, est une boisson dont la technologie a été inspirée d'une recette brésilienne et adaptée au Bénin ; il est obtenu par la cuisson au bain marie pendant environ 3 $\mathrm{h}$ d'un jus naturel de pomme de cajou, précédemment clarifié, mis en bouteille, capsulé et pasteurisé. Au cours de ce traitement thermique appétissant, ce jus subit un brunissement par réaction de Maillard et développe un goût de caramel encore plus agréable que celui du jus naturel.

La vulgarisation de ces procédés mis au point par la recherche est assurée par des ONGs, des structures étatiques telles que les Centres d'Actions Régionales pour le Développement Rural, des partenaires techniques et financiers, à travers des formations périodiques généralement offertes aux groupements et associations menant des activités génératrices de revenus présents dans la zone de production de l'anacardier au Bénin. En effet, l'objectif de ces diffusions des procédés est de mieux valoriser les pommes de cajou au Bénin en vue de réduire les manques à gagner aux producteurs d'anacarde, occasionnés par les pertes postrécolte de cette ressource. Ces formations successives ont suscité la mise en place progressive d'unités de transformation semi artisanales de la pomme de cajou. Ceci aura un impact positif important sur la filière anacarde.

Un total de 10 unités de transformation des pommes de cajou ont été identifiées suite aux entretiens avec les personnes enquêtées. Le Tableau 1 présente la répartition des unités de transformation de la pomme de cajou et les sous produits identifiés par localité.

Le jus de pomme de cajou, en particulier le cajuina, reste le produit de transformation de la pomme de cajou le plus répandu (produit par $80 \%$ des unités); il est suivi de l'alcool, du sirop, de la confiture et du vin de pomme de cajou produits respectivement par $30 \%, 30 \%, 20 \%$ et $10 \%$ des unités. Il a été également constaté que $70 \%$ des unités de transformation de la pomme de cajou au Bénin sont situées dans le département des Collines au centre du Bénin. En effet, les premières formations sur les procédés de transformation de la pomme de cajou notamment en jus de cajou frais et cajuina au Bénin ont été organisées dans ce département des Collines au cours des années 2007 et 2008. C'est seulement en 2013 que les populations du nord-est (départements du Borgou/Alibori), par exemple, ont été en contact avec la technologie de transformation des pommes de cajou en jus. Dans les départements du nord-ouest (Atacora/Donga), les procédés de transformation de la pomme de cajou en jus sont encore méconnus.

$\mathrm{Au}$ Bénin, les principaux atouts de ces nouvelles unités de valorisation de la pomme résident dans la mise au point des procédés, l'appui technique et financier en faveur des unités de transformation, ainsi que l'existence d'une stratégie nationale de promotion de la filière anacarde. Cependant, cette chaîne de valeur, encore embryonnaire, reste globalement sous-développée et confrontée à de nombreuses contraintes, dont l'incapacité des unités à transformer toute la matière première disponible pendant la courte campagne (de janvier à avril), en raison du manque d'équipements appropriés de transformation et d'infrastructures répondant aux normes, les difficultés 
d'approvisionnement en pommes de cajou auprès des producteurs qui privilégient la qualité des noix au détriment de celle des pommes, le nombre limité d'unités de transformation de pommes de cajou et les faibles capacités de production de celles-ci, en dehors d'une seule unité industrielle de production d'alcool alimentaire, installée par l'état dans la commune de Bantè et non encore fonctionnelle.

\section{Systèmes techniques de production du jus de pomme de cajou}

A l'issue de l'enquête, deux procédés de production de jus de pommes de cajou ont été identifiés (le procédé de production du jus naturel de pomme de cajou et celui du cajuina). Quel que soit le procédé utilisé, les fruits sont triés, lavés, découpés et pressés. Le jus brut obtenu est ensuite clarifié par addition d'un agent clarifiant (amidon de manioc ou gruau de riz), puis filtré avant tout traitement thermique (Figure 2).

Le matériel de production utilisé dans les trois unités est composé: d'une presse à vis en acier inoxydable; d'un pasteurisateur en acier inoxydable ; d'un capsuleur mécanique, d'une balance mécanique et du petit matériel (bassines et seaux en plastique utilisés pour le transport, le nettoyage et le lavage des pommes, couteaux en acier inoxydable pour découper les pommes en vue de faciliter le pressage du jus, toiles mousseline servant à filtrer le jus).

La presse à vis en acier inoxydable, de fabrication locale, est formée d'une cage cylindrique perforée pour laisser couler le jus extrait. Cette cage cylindrique repose sur une plaque métallique fixée sur le châssis de la presse. Les pommes découpées et disposées dans l'enceinte de la cage cylindrique sont comprimées entre des rondelles de bois et la plaque métallique à l'aide d'une vis sans fin et le jus sort sous l'effet de la pression ainsi exercée.

Le pasteurisateur comprend une cuve cylindrique dans laquelle repose une plaque circulaire. L'ensemble, surmonté d'un couvercle muni d'un thermomètre pour contrôler la température de l'enceinte et d'une soupape de sécurité permettant d'évacuer le surplus de vapeur, est alimenté par le feu d'un réchaud à gaz.

L'analyse du diagramme de production du jus de pommes de cajou a révélé quelques nuances technologiques au niveau de la clarification et du traitement thermique :

- La clarification se fait de deux façons, qui diffèrent de par le type d'agent clarifiant (amidon de manioc ou gruau de riz) utilisé. Elle comporte pour les deux nuances technologiques, trois principales étapes, à savoir: l'ajout de l'agent clarifiant au jus brut extrait, suivi d'une homogénéisation manuelle du mélange; la sédimentation des particules par décantation du mélange laissé au repos pendant 20 à 120 minutes et enfin la séparation du surnagent par trop plein suivie d'une filtration à travers une toile mousseline qui permet d'éliminer la majorité du reste des particules en suspension et de rendre le jus beaucoup plus limpide.

Pour la clarification à l'amidon de manioc, $5 \mathrm{ml}$ d'amidon liquéfié permettent de clarifier 11 de jus brut après 120 minutes de décantation. L'amidon liquéfié est obtenu par dissolution de $2 \mathrm{~g}$ de poudre d'amidon dans $10 \mathrm{~mL}$ d'eau à $60{ }^{\circ} \mathrm{C}$. Par contre, la clarification au gruau de riz nécessite $125 \mathrm{ml}$ de gruau de riz pour clarifier 11 de jus brut après 20 minutes de décantation. Le gruau de riz est obtenu par cuisson d'un kilogramme de riz dans 61 d'eau pendant 15 minutes. L'ajout d'une si importante quantité de gruau $(12,5 \% \mathrm{v} / \mathrm{v})$ provoque une dilution du jus et une réduction de son goût.

- Le traitement thermique appliqué aux jus varie d'une unité à l'autre. Pour l'obtention du cajuina, les bouteilles contenant du jus de pomme de cajou, préchauffées à $60-70{ }^{\circ} \mathrm{C}$, sont plongées dans une grande marmite d'eau bouillante tapissée de sac de jute. L'ensemble est bouilli à $100{ }^{\circ} \mathrm{C}$ pendant trois heures sur feu de bois de chauffe, selon la pratique observée dans les unités Irédé et Oloré. 
Le jus naturel de pomme de cajou est pasteurisé en vrac à $90{ }^{\circ} \mathrm{C}$ à Jaram avant d'être embouteillé à chaud. En effet, le jus clarifié est progressivement chauffé jusqu'à ce que le thermomètre situé sur la partie supérieure du pasteurisateur indique une température de $90{ }^{\circ} \mathrm{C}$ au bout de 75 minutes en moyenne.

Le cajuina présente un goût de cuit et une couleur jaune foncé différente de celle du jus de pomme naturel qui est beaucoup plus clair.

En somme, tous les procédés utilisés par ces unités comportent un double traitement thermique combinant une pasteurisation et une appertisation. Trois types de jus ont été identifiés; il s'agit: du cajuina clarifié au gruau de riz, du cajuina clarifié à l'amidon de manioc et du jus de pomme naturel clarifié au gruau de riz.

\section{Contraintes technologiques identifiées dans le processus de production des jus de pommes de cajou au Bénin}

Les contraintes identifiées dans le processus de production des jus de pommes de cajou au Bénin sont les suivantes: le faible rendement d'extraction du jus de la presse à vis utilisée, la non maîtrise par les productrices de la durée optimale de clarification et de la dose convenable d'agent clarifiant (amidon de manioc ou gruau de riz) nécessaires pour une bonne élimination des tanins, responsables de l'astringence du jus, la dilution du jus induite par le gruau de riz utilisé pour la clarification et la formation de particules qui se déposent au fond des bouteilles au cours de l'entreposage. A cela s'ajoute la pratique de barèmes inappropriés de traitement thermique du jus naturel, caractérisé par une durée trop longue et une température trop élevée.

\section{Bilan des matières}

Le Tableau 2 présente le bilan des matières au cours de la production des jus de pommes de cajou dans les trois unités enquêtées. On note une perte progressive de matière au fur et à mesure que les opérations unitaires se succèdent. Ainsi, le découpage des pommes de cajou après les étapes de lavage et de triage engendre une perte moyenne de matière de $18,55 \%$. La presse à vis utilisée a une capacité moyenne d'extraction de $51,71 \mathrm{~kg}$ de jus par heure avec un rendement moyen en jus de 60,62\%.

Le gruau de riz permet d'obtenir une masse de jus clair supérieure à celle du jus obtenu avec l'amidon de manioc liquéfié. En effet, le rendement en jus clarifié est de $89,17 \%$ et $83,47 \%$ pour le gruau de riz et l'amidon de manioc respectivement.

\section{Caractéristiques physico-chimiques des jus}

Les Tableaux 3 et 4 présentent les caractéristiques physico-chimiques des boissons. Les trois boissons présentent la même acidité titrable ( $0,31 \%$ d'acide citrique) et un $\mathrm{pH}$ de 3,9 à 4 . La teneur en tanins des trois types de boisson, exprimée en équivalent de catéchine est significativement différente au seuil de 5\%. Elle varie de 4,9 à 14,8 $\mathrm{mg}$ Eq de catéchine/100 ml. Le cajuina clarifié à l'amidon de manioc présente la teneur en tanins la plus élevée. Tandis que le jus clarifié au gruau de riz a la plus faible teneur en tanins. La clarté, la couleur, l'indice de brunissement varient respectivement de 88 à $92 \%, 0,07$ à 0,38 et de 0,12 à 0,32 . Le jus clarifié au gruau de riz est le moins coloré avec l'indice de brunissement le plus faible.

La teneur en vitamine $\mathrm{C}$ des jus varie de 147,07 à $185,79 \mathrm{mg} / 100 \mathrm{ml}$. Les trois boissons contiennent du potassium, du magnésium, du phosphore, du sodium, du fer et du calcium. Le potassium est le minéral dont la teneur est la plus élevée $(145,03$ à $134,26 \mathrm{mg} / 100 \mathrm{~g}$ ) tandis que calcium et le fer ont les plus faibles teneurs (2,01 à 2,09 $\mathrm{mg} / 100 \quad \mathrm{~g} ; \quad 1,54 \quad$ à $\quad 2,97 \quad \mathrm{mg} / 100 \quad \mathrm{~g}$ respectivement).

$\begin{aligned} & \text { Caractéristiques } \\ & \text { boissons }\end{aligned}$
$\begin{array}{lll}\text { Le Tableau } & 5 \text { présente } & \text { les } \\ \text { caractéristiques microbiologiques } & \text { des }\end{array}$


boissons. En dehors du jus de pomme clarifié au gruau de riz où il a été révélé quelques colonies au niveau de la flore aérobie mésophile totale, on note une absence de levures et de moisissures dans toutes les boissons.

\section{Qualité sensorielle des jus}

Le Tableau 6 présente l'appréciation des boissons par les dégustateurs. Les nuances technologiques relatives à l'agent de clarification utilisé (amidon de manioc ou gruau de riz) et au traitement thermique (pasteurisation ou cuisson) ont introduit des différences dans les caractéristiques organoleptiques des boissons.

Par rapport à la couleur, les trois boissons sont significativement différentes ( $p$ $\leq 0,05)$. Les valeurs moyennes des notes accordées par les dégustateurs sont comprises entre 4 (un peu désagréable) et 7 (agréable). Tandis que pour la clarté, les valeurs moyennes des notes accordées par les dégustateurs sont comprises entre 5 (ni agréable ni désagréable) et 7 (agréable).
Aucune différence significative n'a été observée entre les trois boissons $(\mathrm{p} \leq 0,05)$.

En ce qui concerne le goût, le cajuina clarifié au gruau de riz et le cajuina clarifié à l'amidon de manioc ne présentent pas de différence significative $(\mathrm{p} \leq 0,05)$. Mais le jus naturel de pomme de cajou clarifié au gruau de riz est significativement différents des deux autres $(p \leq 0,05)$. Les valeurs moyennes des notes accordées par les dégustateurs sont comprises entre 5 (ni agréable ni désagréable) et 8 (très agréable). Pour l'odeur, les trois boissons sont significativement différentes ( $p$ $\leq 0,05)$. Les valeurs moyennes des notes accordées par les dégustateurs sont comprises entre 5 (ni agréable ni désagréable) et 8 (très agréable).

De façon globale, tous les trois jus sont acceptés par les dégustateurs pour l'ensemble de leurs caractéristiques sensorielles. Mais les deux types de cajuina sont plus appréciés que le jus naturel de pomme cajou. En effet, ces deux types de cajuina ont, selon les dégustateurs, un goût original de caramel et sont très agréables à consommer.

Tableau 1 : Répartition des unités de transformation de la pomme de cajou et les sous produits identifiés par localité.

\begin{tabular}{llccc}
\hline Départements & Communes & Localités & Nombre d'unités & $\begin{array}{c}\text { Sous-produits } \\
\text { identifiés }\end{array}$ \\
\hline Atacora & Kouandé & Kouandé & 1 & Alcool, sirop \\
\hline Borgou & Tchaourou & Boucousséra & 1 & Jus \\
& Parakou & Boco & 1 & Vin, confiture \\
\hline Collines & Dassa-Zoumè & Ayedéro & 1 & Jus \\
& Bantè & Akpassi & 1 & Jus \\
& Bantè & Agoua & 1 & Jus \\
& Savè & Savè & 1 & Jus \\
& Ouèssè & Kilibo & 1 & Jus \\
& Glazoué & Thio & 1 & Jus, confiture, alcool \\
& Glazoué & Gotho & 1 & Jus, confiture, alcool, \\
& & & & sirop \\
\hline
\end{tabular}


Tableau 2 : Bilan des matières au cours de la production du jus de pomme cajou.

\begin{tabular}{lccccc}
\hline \multirow{2}{*}{ Paramètres } & \multicolumn{3}{c}{ Essais } & \multicolumn{1}{c}{ Moyenne } \\
\cline { 2 - 7 } & $\mathbf{1}$ & $\mathbf{2}$ & $\mathbf{3}$ & $\mathbf{4}$ & \\
\hline Masse de pomme cajou utilisée (kg) & 39 & 35 & 38 & 40 & $38 \pm 2,16$ \\
Masse de pomme cajou découpée (kg) & 33 & 28 & 28 & 35 & $31 \pm 3,56$ \\
Perte de matière après découpage (\%) & 15,38 & 20 & 26,32 & 12,5 & $18,55 \pm 6,03$ \\
Masse de jus extrait (kg) & 19,2 & 17,2 & 18 & 20,5 & $18,72 \pm 1,44$ \\
Durée d'extraction (minute) & 22 & 20 & 20 & 25 & $21,75 \pm 2,36$ \\
Capacité d'extraction de la presse (kg/heure) & 52,36 & 51,6 & 54 & 49,2 & $51,79 \pm 2,00$ \\
Rendement en jus (\%) & 58,18 & 61,43 & 64,29 & 58,57 & $60,62 \pm 2,84$ \\
Masse de jus brut utilisée (kg) & 18 & 18 & 18 & 18 & $18 \pm 0,00$ \\
Masse de jus clarifié à l'amidon de manioc obtenu (kg) & 14,6 & 14 & 15 & 16,5 & $15,02 \pm 1,07$ \\
Rendement en jus clarifié à l'amidon de manioc (\%) & 81,11 & 77,78 & 83,33 & 91,67 & $83,47 \pm 5,92$ \\
Masse de jus clarifié au gruau de riz obtenu (kg) & 15,5 & 16,5 & 16,2 & 16 & $16,05 \pm 0,42$ \\
Rendement en jus clarifié au gruau riz (\%) & 86,11 & 91,67 & 90,00 & 88,89 & $89,17 \pm 2,34$ \\
\hline
\end{tabular}

Tableau 3: Caractéristiques physico-chimiques des jus.

\begin{tabular}{lccc}
\hline \multirow{2}{*}{ Paramètres } & \multicolumn{3}{c}{ Echantillons } \\
\cline { 2 - 4 } & $\mathbf{J 1}$ & $\mathbf{J 2}$ & $\mathbf{J 3}$ \\
\hline Acidité titrable $(\%)$ & $0,31 \pm 0,01 \mathrm{a}$ & $0,31 \pm 0,01 \mathrm{a}$ & $0,31 \pm 0,01 \mathrm{a}$ \\
$\mathrm{pH}$ & $4 \pm 0,0 \mathrm{a}$ & $3,9 \pm 0,0 \mathrm{a}$ & $3,9 \pm 0,0 \mathrm{a}$ \\
Tanin (mg Eq de catéchine/100ml) & $14,8 \pm 0,0 \mathrm{a}$ & $5,2 \pm 0,0 \mathrm{~b}$ & $4,9 \pm 0,0 \mathrm{c}$ \\
Extrait Sec Réfractrométrique $(\%)$ & $14 \pm 0,0 \mathrm{a}$ & $11,5 \pm 0,0 \mathrm{~b}$ & $11 \pm 0,0 \mathrm{~b}$ \\
Clarté $\left(\% \mathrm{~T}_{660}\right)$ & $92 \pm 0,0 \mathrm{a}$ & $89,5 \pm 0,0 \mathrm{~b}$ & $88 \pm 0 \mathrm{~b}$ \\
Couleur $\left(\mathrm{A}_{440}\right)$ & $0,36 \pm 0,0 \mathrm{a}$ & $0,38 \pm 0,0 \mathrm{a}$ & $0,069 \pm 0 \mathrm{~b}$ \\
Indice de Brunissement $\left(\mathrm{A}_{420}\right)$ & $0,32 \pm 0,0 \mathrm{a}$ & $0,31 \pm 0,0 \mathrm{a}$ & $0,12 \pm 0 \mathrm{~b}$ \\
Vitamine C (mg/100ml) & $147,0 \pm 0,5 \mathrm{a}$ & $184,6 \pm 0,3 \mathrm{~b}$ & $185,8 \pm 0,2 \mathrm{~b}$ \\
\hline $\begin{array}{l}\text { Les valeurs d'une même ligne affectées de la même lettre sont statistiquement identiques au seuil de } 5 \% \text { selon le test de } \\
\text { Student-Newman-Keuls. }\end{array}$ & & & \\
J1 : Cajuina clarifié à l'amidon de manioc & & & \\
J2 : Cajuina clarifié au gruau de riz & & & \\
J3 : Jus clarifié au gruau de riz & & &
\end{tabular}


Tableau 4: Teneurs de jus de pomme cajou en sels minéraux.

\begin{tabular}{lccc}
\hline & \multicolumn{3}{c}{ Echantillons } \\
\cline { 2 - 4 } Paramètres & $\mathbf{J 1}$ & $\mathbf{J 2}$ & $\mathbf{J 3}$ \\
\hline Calcium $(\mathrm{mg} / 100 \mathrm{~g})$ & $2,12 \pm 0,04 \mathrm{a}$ & $2,00 \pm 0,01 \mathrm{~b}$ & $2,01 \pm 0,01 \mathrm{~b}$ \\
Magnésium $(\mathrm{mg} / 100 \mathrm{~g})$ & $14,05 \pm 0,09 \mathrm{a}$ & $13,41 \pm 0,51 \mathrm{a}$ & $14,84 \pm 0,66 \mathrm{a}$ \\
Potassium $(\mathrm{mg} / 100 \mathrm{~g})$ & $145,03 \pm 3,33 \mathrm{a}$ & $134,26 \pm 2,36 \mathrm{a}$ & $138,95 \pm 0,52 \mathrm{a}$ \\
Sodium $(\mathrm{mg} / 100 \mathrm{~g})$ & $3,01 \pm 0,16 \mathrm{a}$ & $3,02 \pm 0,10 \mathrm{a}$ & $3,55 \pm 0,00 \mathrm{a}$ \\
Phosphore $(\mathrm{mg} / 100 \mathrm{~g})$ & $9,98 \pm 0,47 \mathrm{a}$ & $8,63 \pm 0,29 \mathrm{a}$ & $9,4 \pm 0,07 \mathrm{a}$ \\
Fer (mg/100g) & $2,97 \pm 0,01 \mathrm{a}$ & $2,53 \pm 0,01 \mathrm{a}$ & $1,54 \pm 0,00 \mathrm{~b}$ \\
\hline $\begin{array}{l}\text { Les valeurs d'une même ligne affectées de la même lettre sont statistiquement identiques } \\
\text { au seuil de 5\% selon le test de Student-Newman-Keuls. }\end{array}$ \\
J1 : Cajuina clarifié à l'amidon de manioc & & \\
J2 : Cajuina clarifié au gruau de riz \\
J3 : Jus clarifié au gruau de riz
\end{tabular}

Tableau 5: Caractéristiques microbiologiques des boissons.

\begin{tabular}{lcccc}
\hline Groupe de microorganismes & J1 & J2 & J3 & Normes $^{1}$ \\
\hline Flore totale & - & - & 2 & - \\
Levures & - & - & - & $<20 / \mathrm{L}$ \\
Moisissures & & & & $<10 / 100 \mathrm{ml}$ \\
\hline$\quad$ proposée par Guiraud et Galzy (1980) & & &
\end{tabular}

Tableau 6: Appréciation sensorielle des boissons par les dégustateurs.

\begin{tabular}{lccc}
\hline & \multicolumn{3}{c}{ Echantillons } \\
\cline { 2 - 4 } Paramètres & $\mathbf{J 1}$ & $\mathbf{J 2}$ & $\mathbf{J 3}$ \\
\hline Clarté & $5,8 \pm 0,1 \mathrm{a}$ & $6,4 \pm 0,1 \mathrm{a}$ & $6 \pm 0,0 \mathrm{a}$ \\
Couleur & $5,5 \pm 0,0 \mathrm{~b}$ & $7 \pm 0,1 \mathrm{a}$ & $4,5 \pm 0,1 \mathrm{c}$ \\
Goût & $7,06 \pm 0,0 \mathrm{a}$ & $7,5 \pm 0,1 \mathrm{a}$ & $5 \pm 0,1 \mathrm{~b}$ \\
Odeur & $6 \pm 0,1 \mathrm{~b}$ & $7,5 \pm 0,2 \mathrm{a}$ & $5 \pm 0,1 \mathrm{c}$ \\
Acceptabilité globale & $6 \pm 0,1 \mathrm{~b}$ & $7,1 \pm 0,1 \mathrm{a}$ & $5,1 \pm 0,1 \mathrm{c}$
\end{tabular}

\footnotetext{
Les valeurs d'une même ligne affectées de la même lettre sont statistiquement identiques au seuil de 5\% selon le test de Student-Newman-Keuls.

$\mathrm{J} 1$ : Cajuina clarifié à l'amidon de manioc

J2 : Cajuina clarifié au gruau de riz

$\mathrm{J} 3$ : Jus clarifié au gruau de riz
} 


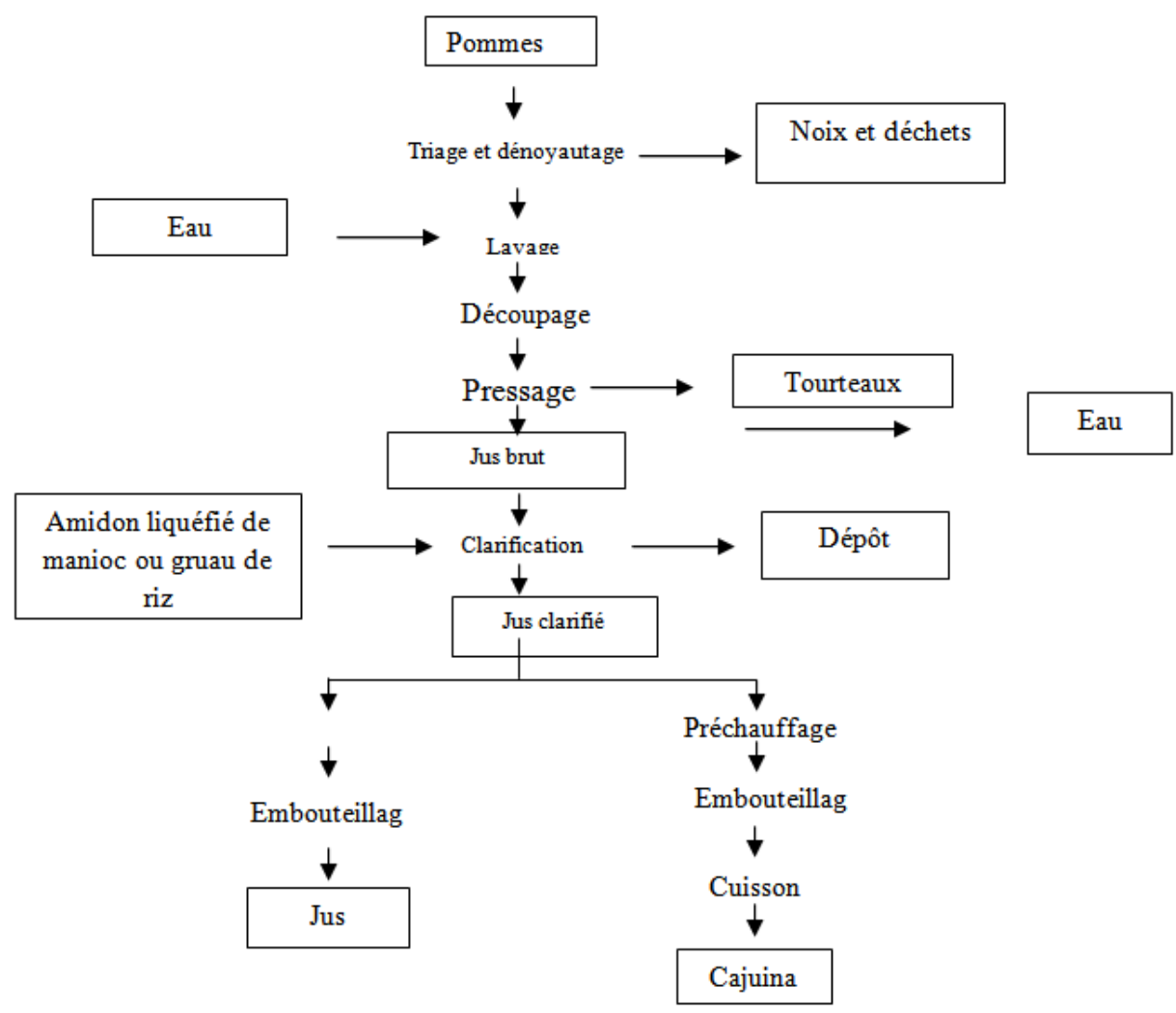

Figure 2: Diagramme technologique de production du jus de pomme cajou et du cajuina.

\section{DISCUSSION}

La pomme de cajou initialement abandonnée au pourrissement dans les plantations d'anacarde gagne de l'importance de nos jours. Les raisons de la faible valorisation de la pomme sont liées à la méconnaissance des technologies de sa transformation et surtout à la rumeur persistante selon laquelle la consommation de la pomme fraîche serait incompatible avec certains produits comme le lait, le sucre et l'alcool à base de vin de palme, connu sous le nom de sodabi au Bénin. Cette hypothèse pourtant non vérifiée a eu des effets négatifs sur la consommation de la pomme et provoqué le rejet de ses sous produits par les populations. Le même constat a été fait par Lacroix (2003). Cependant, divers procédés de valorisation en alcool, jus, vin, confiture et autres ont été élaborés et diffusés. Ces divers procédés ont été également identifiés au Bénin par Kpanou en 2003.

Le rendement moyen en masse de jus brut extrait par rapport aux pommes est de $60,62 \%$. Ce résultat se justifie par la faible performance de la presse à vis utilisée, mais aussi par le découpage grossier de la pomme. En effet, en raison du faible taux d'extraction du jus, une quantité non négligeable de jus est encore retenue par le tourteau après le pressage. Ce rendement de la presse à vis est moins important que celui obtenu par Akinwale et al. (2001) à l'aide d'un extracteur de jus de type expeller qui est de $67,88 \%$. La clarification avec les différents agents a entraîné des pertes en jus considérables (8 à $22 \%$ ). Plus le dépôt après décantation est important, plus les pertes sont abondantes. En effet, le dépôt est composé de jus et de petits flocons engendrés par la réaction entre les 
substances chimiques du jus de pomme cajou et l'agent de clarification (da Silva Neto et al., 2009).

La durée et la température des traitements thermiques affectent la clarté, la couleur, l'indice de brunissement et la teneur en tanins des jus. Le chauffage du jus pendant un temps relativement long engendre des jus beaucoup plus sombres. La même observation a été faite par Damasceno et al. (2008) au cours du traitement thermique du cajuina à 80 et $121{ }^{\circ} \mathrm{C}$. Pour ces auteurs, la perte en acide ascorbique au cours du traitement thermique pourrait être le principal facteur responsable du brunissement du jus. Selon Akinwalé et al. (2001), l'augmentation de l'indice de brunissement serait due à la caramélisation des sucres du jus. Le jus clarifié au gruau de riz est le plus clair de tous les jus, en ce sens qu'il a été dilué lors de la clarification et n'a pas subi de cuisson. La couleur, la clarté, les indices de brunissement du cajuina clarifié au gruau de riz et du cajuina clarifié à l'amidon de manioc sont statistiquement identiques. Les valeurs de la clarté des trois boissons sont comprises dans la gamme de celles $(88,53$ à $98,0 \%$ ) obtenues par Talasila et al. (2012a) pour les jus de pommes de cajou brut et clarifié.

Le jus naturel de pommes de cajou a une teneur en extrait sec réfractométrique de $11{ }^{\circ}$ Brix pendant que le cajuina clarifié au gruau de riz et celui clarifié à l'amidon de manioc ont une teneur en extrait sec réfractométrique de 11,5 et de $14{ }^{\circ}$ Brix respectivement. Cette faible teneur en matière sèche soluble du jus clarifié au gruau de riz et du cajuina clarifié au gruau de riz serait due à la réduction de la concentration de ces jus par l'ajout du gruau de riz, comme l'ont indiqué Dossou et al. (2008) à l'élaboration du procédé au Bénin. Akinwalé (2001) au Nigéria et Adou et al. (2012) en Côte d'ivoire ont obtenu respectivement une teneur en extrait sec réfractométrique de 11 et 10,2 à $10,9{ }^{\circ}$ Brix pour le jus brut de pommes cajou. Ces valeurs ne sont pas identiques à celles trouvées dans la présente étude. Ceci pourrait s'expliquer par des différences de conditions pédologiques et climatiques de ces pays.

Les trois boissons de pommes de cajou analysées ont un $\mathrm{pH}$ compris entre 3,9 et 4 avec une acidité de 0,31\%. Akinwalé (2000), Damasceno et al. (2008) et Adou et al. (2012) ont obtenu respectivement un $\mathrm{pH}$ de 4,15 ; 4,$4 ; 4,37$ à 4,5 pour des jus brut de pomme de cajou. Akinwalé en 2000 a obtenu une acidité titrable de $0,48 \%$ contre 0,55 à $0,59 \%$ pour le jus de pomme de cajou mûre selon les travaux de Adou et al. (2012). Cette différence pourrait s'expliquer par le fait que ces auteurs ont travaillé sur des jus bruts (non clarifiés) de cajou d'une part et d'autre part, Adou et al. (2012) ont utilisé des pommes de différentes variétés, provenant de deux différentes régions de la Côte d'Ivoire. Plusieurs facteurs, notamment la variété, la zone agroécologique, le climat, les pratiques culturales, le niveau de maturité du fruit à la récolte (Drake et Eisele, 1994), les conditions de stockage du fruit (Drake et al., 2002; Drake et Eisele, 1999) et même les couleurs et formes des pommes cajou affectent leur composition physico-chimique (Adou et al., 2012).

La teneur en vitamine $\mathrm{C}$ des jus qui varie de 147,07 à $185,79 \mathrm{mg} / 100 \mathrm{ml}$ est comprise dans la gamme des valeurs obtenues par Akinwalé et al. (2001) au Nigéria après 10 à 30 minutes de chauffage des pommes de cajou qui est de 120,60 à $152,40 \mathrm{mg} / 100 \mathrm{ml}$ contre $195,80 \mathrm{mg} / 100 \mathrm{ml}$ pour le jus brut de pommes de cajou non chauffées, tandis que Talasila et al. (2012b) ont obtenu pour le jus de pomme de cajou 123,9 à 184,46 mg/100 ml pour la vitamine $\mathrm{C}$.

Lowor (2009) a déterminé pour des jus bruts extraits de différentes variétés de pommes de cajou du Ghana une teneur en potassium de $76,0 \mathrm{mg} / 100 \mathrm{ml}$, suivie du calcium $(43,0 \mathrm{mg} / 100 \mathrm{ml})$, du magnésium $(10,92 \mathrm{mg} / 100 \mathrm{ml})$, du phosphore $(0,79$ $\mathrm{mg} / 100 \mathrm{ml})$ et du sodium $(0,41 \mathrm{mg} / 100 \mathrm{ml})$. Adou et al. (2011) en Côte d'ivoire ont obtenu pour des jus bruts de pomme de cajou une teneur en potassium de 204,38 à 218,9 $\mathrm{mg} / 100 \mathrm{ml}$, suivie du phosphore $(21,13$ à 
23,97 mg/100ml), du magnésium $(15,29$ à $21,51 \mathrm{mg} / 100 \mathrm{ml}$ et du sodium $(3,39$ à 4,33 $\mathrm{mg} / 100 \mathrm{ml})$. La teneur en potassium est la plus élevée $(145,03$ à 134,26 mg/100g) dans les trois boissons du Bénin tandis que leurs teneurs en calcium et en fer $(2,01$ à 2,09 $\mathrm{mg} / 100 \mathrm{~g} ; \quad 1,54 \quad$ à $\quad 2,97 \quad \mathrm{mg} / 100 \mathrm{~g}$ respectivement) sont les plus faibles. Les teneurs en calcium et en phosphore de ces différentes boissons sont comprises dans la gamme des valeurs obtenues par l'USDA (2002) qui varient respectivement de 0,9 à 5,4 $\mathrm{mg} / 100 \mathrm{~g}$ et de 6 à $21 \mathrm{mg} / 100 \mathrm{~g}$ pour la pomme de cajou. La teneur en fer des jus qui est comprise entre 1,54 et $2,97 \mathrm{mg} / 100 \mathrm{~g}$ est supérieure à celle indiquée par l'USDA (2002) qui est de 0,19 - 0,71 mg/100g de pomme de cajou alors que, selon la même source, les teneurs en potassium et en magnésium $(565 \mathrm{mg} / 100 \mathrm{~g}$ et $260 \mathrm{mg} / 100 \mathrm{~g}$ respectivement) de la pomme de cajou sont supérieures à celles des jus analysés dans la présente étude. Cette variation dans la composition minérale pourrait s'expliquer par la variation des conditions pédologiques et des pratiques culturales d'une localité à une autre. Les minéraux sont des micronutriments qui jouent un rôle important dans les processus métaboliques du corps humain. $\mathrm{La}$ consommation des fruits et légumes améliore la régulation de la teneur en minéraux de l'organisme et réduit le risque de maladies cardiovasculaires et de cancer (Ismail et al., 2011). Les groupes vulnérables que sont les femmes enceintes, les femmes allaitantes et les enfants sont les premières victimes des carences en micronutriments en raison de leurs besoins plus importants (Black et al., 2003). En effet, les fruits et légumes sont les meilleures sources de micronutriments (vitamines et minéraux) pour leur organisme (Wargovich, 2000). Le jus de pomme de cajou est une bonne source de minéraux. Les valeurs obtenues pour le calcium $(2,01-2,12 \mathrm{mg} / 100 \mathrm{~g})$ et le fer $(2,97$ $1,54 \mathrm{mg} / 100 \mathrm{~g}$ ) sont supérieures aux apports journaliers recommandés pour ces deux minéraux qui sont de $0,68 \mathrm{mg}$ et $0,015 \mathrm{mg}$ respectivement (USDA, 2002). Le potassium (K) participe au maintien de l'équilibre des électrolytes dans le corps humain et prévient la déminéralisation des os en empêchant la perte de calcium dans les urines (Tucker et al., 1999; He and MacGregor, 2001). Il est un hypotenseur et intervient également dans la contraction musculaire (Appel, 1999). Le magnésium joue un rôle important dans la stabilité du système nerveux, dans la contraction musculaire comme un activateur de la phosphatase alcaline (Cowan, 2002; Ismail et al., 2011). Le sodium (Na) régule la pression artérielle et osmotique. C'est un cation extracellulaire important qui stabilise le fluide extracellulaire (Sagnella et al., 1989). Le phosphore (P) est un constituant des os et des dents où il est combiné avec des ions calcium. Le phosphore participe également à la formation des molécules de base telles que les acides nucléiques (ADN et ARN), l'adénosine triphosphate (ATP) et les phospholipides des membranes (Jacotot and Parco, 2000). Le fer est un composant essentiel de l'hémoglobine qui intervient dans le transport de l'oxygène. En dehors des minéraux, le jus de pomme de cajou est riche en vitamine $\mathrm{C}$ et polyphénols. Ainsi, le jus de pomme de cajou est généralement utilisé pour améliorer la qualité nutritionnelle des jus d'autres fruits tropicaux de faible teneur en vitamine $\mathrm{C}$ comme la mangue et l'ananas (Akinwale, 2000).

Les résultats de l'analyse microbiologique des trois types de boissons montrent que les conditions du milieu sont défavorables au développement des microorganismes. Ceci pourrait s'expliquer par le traitement thermique sévère appliqué à ces jus, mais aussi, Ranganna (1986) fait observer que la plupart des bactéries ne se développent pas aux $\mathrm{pH}$ bas, comme c'est le cas dans les jus analysés dans la présente étude. La même observation a été faite par Talasila et al. (2011).

La couleur du cajuina clarifié au gruau de riz a été la plus appréciée par les dégustateurs. En effet, pour ceux-ci, elle n'est 
ni trop pâle ni foncée et attire le consommateur. Par contre, le cajuina clarifié à l'amidon de manioc qui a une couleur trop foncée et le jus naturel de pomme cajou dont la couleur est trop pâle selon les dégustateurs, sont moins appréciés. Cependant, la mesure de la couleur au spectrophotomètre a donné des valeurs qui ne présentent pas de différence significative au seuil de $5 \%$ pour les deux types de cajuina. En ce qui concerne l'odeur, le jus clarifié au gruau de riz a une odeur moyenne caractéristique de la pomme de cajou, tandis que les deux types de cajuina ont une odeur forte caractéristique de la pomme de cajou et de caramel, encore plus accentuée au niveau du cajuina clarifié à l'amidon de manioc. En effet, l'odeur modérée de pomme de cajou présentée par le jus de pomme de cajou clarifié au gruau de riz serait due à la perte en composés aromatiques de la pomme de cajou lors de la pasteurisation en vrac du jus avant l'embouteillage, tel que l'ont aussi fait remarquer Reineccius et al. (2006). Le goût original de caramel des deux types de cajuina serait dû à la longue durée du traitement thermique qui a conduit à des réactions de Maillard comme l'ont aussi fait savoir Akinwalé et al. (2001).

\section{Conclusion}

L'étude diagnostique des technologies de transformation de la pomme de cajou en jus a permis de disposer de connaissances scientifiques sur les procédés disponibles au Bénin. Ceci permettra d'améliorer globalement ces procédés pour sauvegarder les qualités nutritionnelle, microbiologique et sensorielle des jus. Les contraintes relevées lors de l'enquête étant : (1) le faible rendement d'extraction du jus de presse à vis utilisée (2) la non maîtrise par les productrices de la durée optimale de clarification et de la dose convenable d'agent clarifiant (amidon de manioc ou gruau de riz) nécessaires pour une bonne élimination des tanins, responsables de l'astringence du jus, (3) la dilution du jus induite par la clarification au gruau de riz et (4) la formation de particules qui se déposent au fond des bouteilles au cours de l'entreposage, (5) les mauvaises pratiques de pasteurisation du jus naturel, les améliorations du procédé de production du jus de pomme de cajou au Bénin doivent cibler ces différents aspects. Cette transformation encore embryonnaire fournit des jus ayant de bonnes caractéristiques physico-chimiques et microbiologiques avec une qualité organoleptique globalement appréciée par les dégustateurs. Cependant, puisque la rumeur selon laquelle la consommation de la pomme fraîche serait incompatible avec certains produits comme le lait, le sucre et le sodabi persiste et met en cause la qualité sanitaire de ce fruit, il serait intéressant d'infirmer ou de confirmer cette rumeur en déterminant les propriétés toxicologiques des composés issus de la combinaison du jus de pomme de cajou des variétés d'anacarde du Bénin avec ces produits alimentaires incriminés.

\section{REMERCIEMENTS}

Les auteurs remercient sincèrement les autorités rectorales de l'Université d'AbomeyCalavi pour le soutien financier dans la réalisation de ce travail.

\section{REFERENCES}

Adou M, Tetchi FA, Gbané M, Kouassi KN, Amani NG. 2012. Physico-chemical characterization of cashew apple juice (Anacardium occidentale L.) from yamoussoukro (côted'ivoire). Innov. Rom. Food Biotechnol., 11: 32-43.

Akinwale TO. 2000. Cashew apple juice: Its use in fortifying the nutritional quality of some tropical fruits. Eur. Food Res. Technol., 211: 205-207.

Akinwalé TO, Aladesua OO. 2001. Comparative study of physico-chemical properties and effect of different techniques on the quality of cashew juice from Brazilan and local varieties. Nig. J. Tree Crop Research., 3(1): 56-58.

Appel LJ. 1999. Nonpharmacologic therapies that reduce blood pressure: a fresh perspective. Clin. Card., 22: 1-5. 
Black RE, Morris SS and Bryce J. 2003. Where and why are 10 million children dying every year? Lancet, 361: 2226-2234.

Broadhurst RB, Jones WT. 1978. Analysis of condensed tannins using acidified vanillin. J. Sci. Food Agric., 29: 788-794.

Castro TR, de Abreu FAP, Carioca JOB. 2007. Obtenção de suco clarificado de caju (Anacardium occidentali, L) utilizando processos de separação por membranas. Rev. Ciênc. Agronômica, 38(2): 164-168.

Campos DCP, Santos AS, Wolkoff DB, Matta VM, Cabral LMC, Couri S. 2002. Cashew apple juice stabilization by microfiltration. Desalination, 148(1-3): 61-65.

Cianci FC, Silva LFM, Cabral LMC, Matta VM. 2005 .Clarificação e concentração de suco de caju por processos com membranas. Ciênc. Tecnol. Aliment. Campinas., 25(3): 579-583.

Couri S, Ferreira de Menezes L, Saavedra Pinto G A, de Souza L M; Pereira Freitas S. 2003. Comparison of the cashew apple (Anacardium occidentale L.) juice clarification with tannase and gelatin. Bol. Centro de Pesquisae Processamento de Alimentos., 20(1): 41-54.

Cowan JA. 2002. Structural and catalytic chemistry of magnesium-dependent enzymes. Biometals, 15: 225-235.

Damasceno LF, Fernandes FANM, Brito ES. 2008. Evaluation and optimization of non enzymatic browning of "cajuina" during thermal treatment. Bra. J. Chem Eng., 25(02): 313-320.

da Silva Neto RM, de Abreu FAP, de Assis Paiva FF. 2009. Processamento do Pedúnculo de Caju: Cajuína. Embrapa Agroindústria Tropical Fortaleza, Dezembro. Genervo, 38p.

de Abreu F, Perez AM, Dornier M, Reynes M. 2005. Application of cross-flow microfiltration in the production of clarified cashew juice by means of mineral membranes. Fruits., 60(01): 3340.

Dossou J, Boni P, Cormier R. 2008. Clarification du jus de pomme de cajou. Anacardiuminfo.com

Drake SR, Eisele TA. 1994. Influence of harvest date and controlled atmosphere storage delay on the color and quality of 'delicious' apples stored in a purge-type controlled-atmosphere environment. Hort. Technol., 4: 260-263.

Drake SR, Eisele TA. 1999. Carbohydrate and acid contents of gala apples and bartlett pears from regular and controlled atmosphere storage. J. Agric. Food Chem., 47: 3181-3184.

Drake SR, Elfving DC, Eisele TA. 2002. Harvest maturity and storage affect quality of 'cripps pink' (pink lady) apples. Hort. Technol., 12: 388-391.

FAO (Food and Agriculture Organization). 2014. Base des données de la FAO 2011. http://faostat3.fao.org. Visité le 4 Septembre 2014 à 13 heures.

Guiraud J, Galzy P. 1980. L'Analyse Microbiologique dans les Industries Alimentaires. Collection Génie Alimentaire. Les Editions de l'Usine Nouvelle : Paris ; 240 p.

He FJ, MacGregor GA. 2001. Beneficial effects of potassium. Br. Med. J., 323: 497-501.

IRD (International Relief Service). 2012. The cashew cookbook: Recipes from the Gambia and Senegal, IRD, 42p. africancashewalliance.com

Ismail F, Anjum MR, Mamon AN, Kazi TG. 2011. Trace metal contents of vegetables and fruits of Hyderabad retail market. Pak. J. Nutr., 10: 365-372.

Jacotot B, Le Parco J-C. 2000. Nutrition et Alimentation. Masson: Paris; 311.

Jayalekshmy VG, John PS. 2004. Sago'- a natural product for cashew apple juice clarification. J. Trop. Agric., 42: 67-68.

Kpanou M. 2003. Fabrication de l'alcool à partir de la pomme de cajou. Article $\mathrm{N}^{0}$ 1537, CTA, 11p. 
Lacroix E. 2003. Les anacardiers, les noix cajou et la filière anacarde à Bassila et au Bénin. Térra Systems, 47 p.

Lowor ST, Agyente-Badu CK. 2009. Mineral and proximate composition of cashew apple (Anarcadium occidentale L.): Juice from Northern Savannah, Forest and Coastal Savannah Regions in Ghana. Am. J. Food Technol., 4: 154-161.

Meilgaard M, Civille GV, Carr BT. 1987. Sensory Evaluation Techniques (vol. II). CRC Press: Boca Raton FL.

Michodjehoun-Mestres L, Souquet JM, Fulcrand H, Bouchut C, Reynes M, Brillouet JM. 2009. Monomeric phenols of cashew apple (Anacardium occidentale L.). Food Chem., 112: 851-857.

Queiroz C, Lopes MLM, Fialho E, ValenteMesquita VL. 2011. Changes in bioactive compounds and antioxidant capacity of fresh-cut cashew apple. Food Res. Int., 44: 1459-1462.

Ranganna S. 1986. Handbook of Analysis and Quality Control for Fruit and Vegetable Products (2nd edn). New Delhi: McGraw Hill; 9-80.

Reineccius G. 2006. Changes in Flavor Due to Processing, in Flavor Chemistry and Technology. Reineccius G, Taylor, Francis (eds). Boca Raton, FL; 103-137.

Sagnella GA, Markandu ND, Buckley MG, Miller MA, Singer DR, MacGregor GA. 1989. Hormonal responses to gradual changes in dietary sodium intake in humans. Am. J. Physiol. Regul. Integr. Comp. Physiol., 256: 1171-1175.

Sampaio KL, Garruti DS, Franco MRB, Janzanttic NS, da Silva MAA P. 2011.
Aroma volatiles recovered in the water phase of cashew apple (Anacardium occidentale L.) juice during concentration. J. Sci. Food Agric., 91: 1801-1809.

Sidel JL, Stone H. 1993. The role of sensory evaluation in the food industry. Food Quality and Preference, 4: 65-73.

Talasila U, Vechalapu RR, Shaik KB. 2011. Preservation and shelf life extension of cashew apple juice. Int. J. Food Safety, 13: $275-280$.

Talasila U, Vechalapu RR, Shaik KB. 2012. Clarification, Preservation, and Shelf Life Evaluation of Cashew Apple Juice. Food Sci. Biotechnol., 21(3): 709-714.

Talasila U, Vechalapu RR, Shaik KB. 2012. Storage, stability of cashew apple juiceUse of chemical preservatives. J. Food Technol., 10(4): 117-123.

Tucker KL, Hannan MT, Chen H, Cupples LA, Wilson PWF, Kiel DP. 1999. Potassium, magnesium and fruit and vegetable intakes are associated with greater bone mineral density in elderly men and women. Am. J. Clin. Nutr., 69: 727-36.

USDA. 2002. Nutrition Database for Standard Reference. USDA: Washington.

Wargovich MJ. 2000. Anticancer properties of fruits and vegetables. Hort. Sci., 35: 573575.

Zepka LQ, Mercadante AZ. 2009. Degradation compounds of carotenoids formed during heating of a simulated cashew apple juice. Food Chem., 117: 2834. 